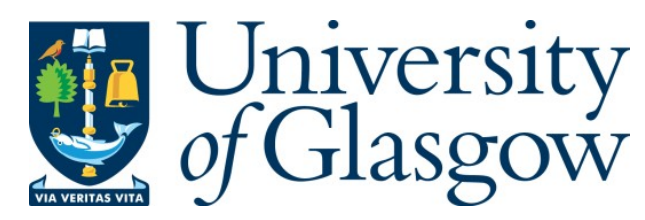

Pusino, V., Strain, M. J., and Sorel, M. (2014) Passive mode-locking in semiconductor lasers with saturable absorbers bandgap shifted through quantum well intermixing. Photonics Research, 2(6), pp. 186-189.

Copyright @ 2014 Chinese Laser Press

Version: Published

$\underline{\text { http://eprints.gla.ac.uk/106494 }}$

Deposited on: 21 May 2015

Enlighten - Research publications by members of the University of Glasgow http://eprints.gla.ac.uk 


\title{
Passive mode-locking in semiconductor lasers with saturable absorbers bandgap shifted through quantum well intermixing
}

\author{
Vincenzo Pusino, ${ }^{1, *}$ Michael J. Strain, ${ }^{1,2}$ and Marc Sorel ${ }^{1}$ \\ ${ }^{1}$ School of Engineering, University of Glasgow—Rankine Building, Oakfield Avenue, Glasgow G128LT, UK \\ ${ }^{2}$ Institute of Photonics, University of Strathclyde, Glasgow G4 ONW, UK \\ ${ }^{*}$ Corresponding author: Vincenzo.Pusino@glasgow.ac.uk
}

Received August 14, 2014; revised October 23, 2014; accepted October 23, 2014; posted November 5, 2014 (Doc. ID 216715); published November 26, 2014

\begin{abstract}
Passive mode-locking in semiconductor lasers in a Fabry-Perot configuration with a bandgap blueshift applied to the saturable absorber (SA) section has been experimentally characterized. For the first time a fully post-growth technique, quantum well intermixing, was adopted to modify the material bandgap in the SA section. The measurements showed not only an expected narrowing of the pulse width but also a significant expansion of the range of bias conditions generating a stable train of optical pulses. Moreover, the pulses from lasers with bandgap shifted absorbers presented reduced chirp and increased peak power with respect to the nonshifted case. (C) 2014 Chinese Laser Press

OCIS codes: $\quad$ (140.4050) Mode-locked lasers; (250.5960) Semiconductor lasers; (130.3120) Integrated optics devices.

http://dx.doi.org/10.1364/PRJ.2.000186
\end{abstract}

\section{INTRODUCTION}

The generation of high frequency, high power optical pulses is of importance for a number of applications, from frequency conversion based on nonlinear phenomena to regeneration of optical pulse trains, as well as terahertz generation [1,2]. Monolithic semiconductor mode-locked lasers (SMLLs), based on the so-called split-contact Fabry-Perot cavity configuration [3] and including a saturable absorber (SA) as a trigger for pulsed operation, are ideal candidates for generation of pulses with high repetition rates because of their very short cavity allowing pulse train frequencies of several tens of gigahertz or higher. However, major drawbacks of SMLLs which are effectively limiting their widespread diffusion and use in commercial applications are the limited average output powers and the narrow biasing parameter range over which stable mode-locking (ML) operation occurs [4].

The analysis in [5] suggested that such limitations are caused by the wavelength-dependent properties of the absorbing section. Starting from a situation of stable and complete ML (as defined in [6]), an increase of the gain section current directly translates into an increase of the SA photocurrent, with the associated Joule heating shifting the SA band edge away from that of the gain section, and the optimal conditions for ML ceasing.

Based on this qualitative analysis, a blue bandgap shift applied to the SA has the potential to shift the range of bias conditions providing stable ML to higher currents. Previous research [7,8] on bandgap-shifted absorber ML showed a pulse width reduction. Here we show that applying a blue detuning to the SA of a split-contact passively mode-locked semiconductor laser in a Fabry-Perot configuration not only narrows the pulse but also reduces the chirp and leads to a great expansion of the range of bias conditions that provide stable ML operation.

The bandgap of the SA region was blueshifted through quantum well intermixing (QWI). Unlike the techniques previously employed to achieve blue detuning of the SA $[7,8]$, QWI is fully post-growth; therefore it provides a simple and low-cost way to effectively induce an area-selective blue detuning on several multi-quantum well (MQW), quantum dot, and quantum dash material systems [9].

\section{DEVICE DESIGN AND FABRICATION}

The devices characterized were fabricated at the University of Glasgow, exploiting the facilities of the James Watt Nanofabrication Centre. The material used was a MQW AlGaInAs/InP wafer structure, and a detailed description of the epitaxial layers can be found in [10]. This particular material was chosen because its ML behavior is well understood and documented, thanks to extensive characterization carried out previously [6]. Besides the insertion of a preliminary QWI step, the fabrication followed a standardized laser fabrication process, whose details can be found elsewhere [11]. The applied bandgap blueshift through QWI had to be carefully chosen, given the lack of extensive published data on the behavior of monolithic SMLLs with blue-detuned absorbers. In [7] a pulse width reduction by more than a factor of two in comparison with a nondetuned SA was reported, from 2.6 to $1.2 \mathrm{ps}$ with a $24 \mathrm{~nm}$ blue detuning. The interpretation given to this result was that, due to the increased transparency of a blue-detuned absorber, a larger reverse bias is required, and therefore the SA has a faster loss recovery. However, excessive blue detuning translates into the requirement for a large SA reverse bias, which might eventually cause a 


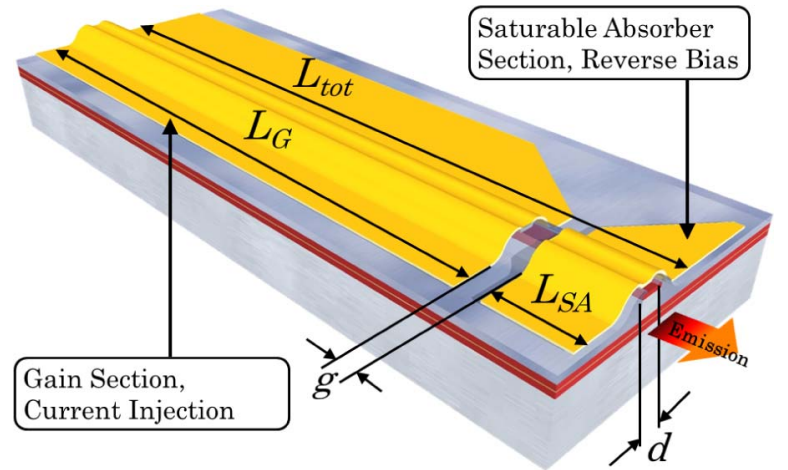

Fig. 1. Schematic of the fabricated device geometry.

junction breakdown because of the high electric field. In view of the previous considerations, the bandgap shift applied to the SA was chosen to be $10 \mathrm{~nm}$.

The geometry of the fabricated structures is depicted in Fig. 1. The sample contained lasers with intermixed SAs and lasers whose absorbers were not blueshifted. For all fabricated SMLLs the length of the absorber section $L_{\mathrm{SA}}$ varied between $1 \%$ and $15 \%$ of the total cavity length $L_{\text {tot }}$, which was chosen to be $1250 \mu \mathrm{m}$ in order to produce optical pulses with a repetition rate of quasi- $40 \mathrm{GHz}$. A $10 \mu \mathrm{m}$ wide gap $g$ between the SA and gain section provided electric insulation, and the laser's waveguide width $d$ was chosen to be $2 \mu \mathrm{m}$ to ensure single-mode propagation.

Prior to the characterization of the SMLLs, the obtained bandgap shift was verified through wavelength scan measurements of a specifically designed waveguide array on a chip, using the setup depicted in Fig. 2(a). The bandgap shift check confirmed the value of $10 \mathrm{~nm}$ which had been targeted, as Fig. 2(b) shows.

\section{EXPERIMENTAL RESULTS AND DISCUSSION}

For the characterization of the devices, the SMLLs were temperature controlled and kept at $20^{\circ} \mathrm{C}$ throughout the whole of the experiments, and their output was collected from the gain section side with an optical fiber lens and simultaneously distributed through fused fiber splitters between an intensity autocorrelator (IAC) and an optical spectrum analyzer (OSA), with power splitting ratios of $90 \%$ and $10 \%$, respectively. Before coupling into the IAC, the optical signal was amplified by means of an erbium-doped fiber amplifier, whose gain was kept constant at $15 \mathrm{~dB}$ and whose output polarization was controlled to maximize the IAC output. A LabView code was used for automating the data collection, for simultaneous

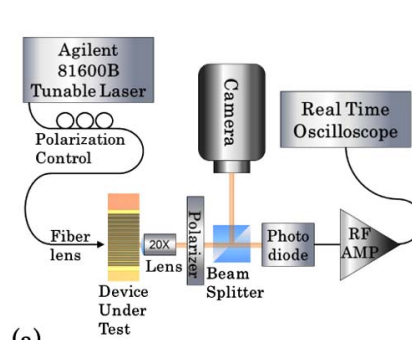

(a)

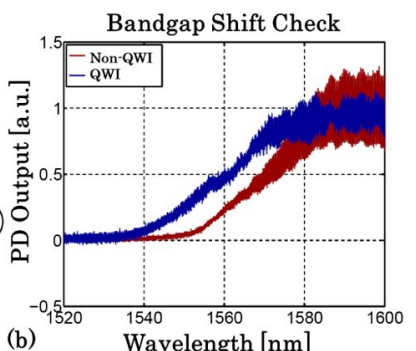

Fig. 2. (a) Schematic of the setup used to test the obtained bandgap shift and (b) band edge comparison between nonintermixed and intermixed cases for $2 \mu \mathrm{m}$ wide waveguides.
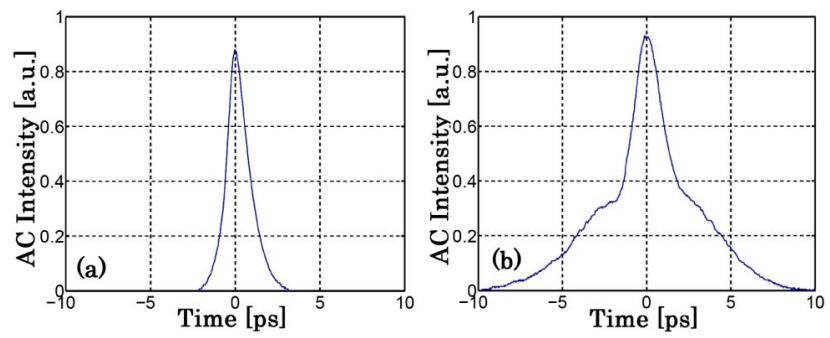

Fig. 3. Comparison between pulses with FWHM of (a) 1.4 ps and (b) $2.7 \mathrm{ps}$ for a SMLL whose blue-detuned SA is $7 \%$ of the total cavity length.

acquisition of the IAC and OSA traces, and for characterization of an extensive range of gain section currents and SA reverse biases.

Devices with SA lengths ranging from $1 \%$ to $7 \%$ of the total cavity length $L_{\text {tot }}$ all produced stable pulse trains, independently of the absorber being blueshifted or not. The IAC data were post-processed in order to extract the full width at halfmaximum (FWHM) of the pulse. This parameter was used to assess the interval of currents and voltages providing stable ML, with a chosen 2.5 ps boundary value since wider pulses were not pedestal-free and displayed dynamical instabilities. A comparison between pulses inside and outside the optimal ML region is presented in Fig. 3. The extension of parameter space producing stable ML increased proportionally to the absorber length, as Fig. 4 illustrates by showing the comparison of the regions where pulses are found to have FWHM of less than $2.5 \mathrm{ps}$ for SMLLs with different SA lengths (1\% and $2 \%$ of the total cavity length). The SMLL with absorbing length of $7 \%$ was the one producing narrow pulses (below $2.5 \mathrm{ps}$ ) over the largest range of operating conditions, for both intermixed and nonintermixed SAs. For lasers with longer absorbing sections, $Q$-switching instability regimes were dominant, in line with expectations [12]. Although the following analysis will focus on the device with a $7 \%$ absorber, it is worth mentioning that the differences due to QWI which will be discussed have been observed also for shorter SAs.

The effect of blue detuning of the SA can be clearly observed from the juxtaposition of the color maps shown in Fig. 5. The maps have been plotted for the same current and voltage intervals and on the same color scale for ease of comparison. It can be seen that the SMLL with intermixed SA [Fig. 5(b)] produces pulses under 2.5 ps to currents nearing $300 \mathrm{~mA}$, whereas the nonintermixed SA emits pulses narrower than $2.5 \mathrm{ps}$ only up to $215 \mathrm{~mA}$ [Fig. 5(a)]. The shortest measured FWHM of the pulses also displays a net improvement, with 0.7 ps obtained for the intermixed case and 1 ps for the nonintermixed one. As for the range of currents and
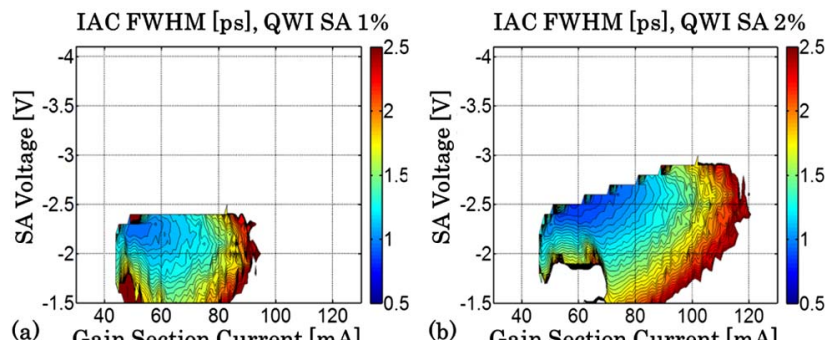

Fig. 4. Color maps show the FWHM of the IAC pulses emitted by the SMLLs with intermixed SAs for absorbers whose length is (left) $1 \%$ of the cavity length and (right) $2 \%$. 

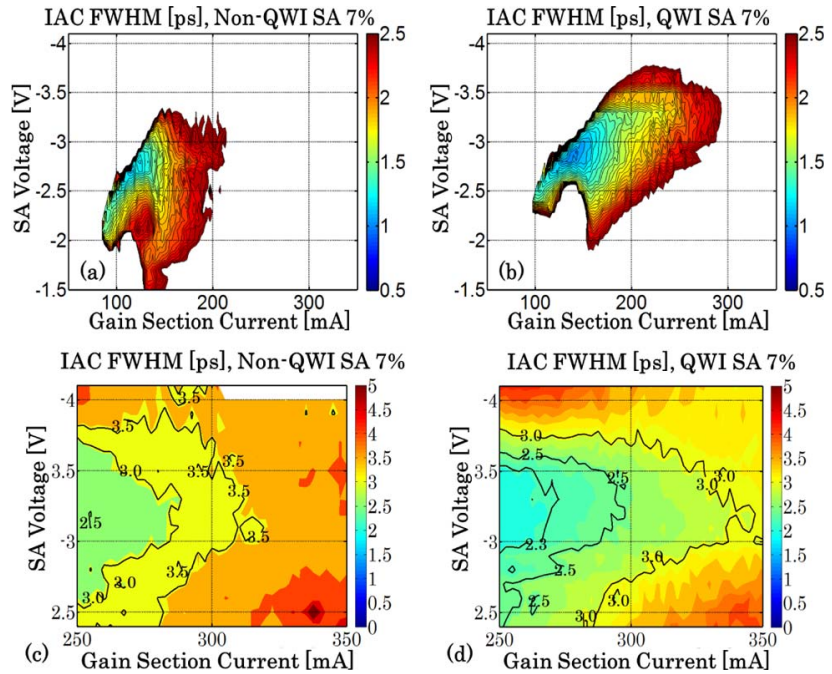

Fig. 5. Color maps show the areas where the FWHM of the IAC pulses is lower than 2.5 ps for (a) one SMLL with nonintermixed $7 \%$ SA and (b) a device with a blue-detuned 7\% SA; (c) and (d) show an enlarged section of the maps for currents between 250 and $350 \mathrm{~mA}$.

voltages providing the narrowest pulses, there is a shift of $0.5 \mathrm{~V}$ toward more negative biases for the QWI case, in line with expectations.

The FWHM of the optical spectrum was also extracted through data post-processing, and the results were combined with the IAC FWHM data to provide the time-bandwidth product (TBP) of the pulses. This quantity tells how close a pulse is to its transform limit, that is, the narrowest possible spectrum for a given pulse duration.

The TBP of a transform-limited sech ${ }^{2}$ pulse, the temporal shape that better fits mode-locked emission from the MQW material used, is 0.315 [13]. Higher values of TBP indicate a chirped pulse. The comparison of the TBP data for the nonintermixed and QWI case indicate that the QWI device exhibits lower chirp, despite both devices being far from the transform limit (Fig. 6). It is thought the chirp reduction is a consequence of complex dispersion and nonlinearity dynamics in both the SA and the gain section, although to confirm this further investigation is required.

The total power versus current measurements, carried out with a broad area detector, showed the same level of average power between intermixed and nonintermixed devices fabricated on the same chip. This together with the narrower pulses leads the intermixed devices to exhibit higher peak power, with $215 \mathrm{~mW}$ achieved versus only $182 \mathrm{~mW}$ obtained from the nonintermixed lasers.
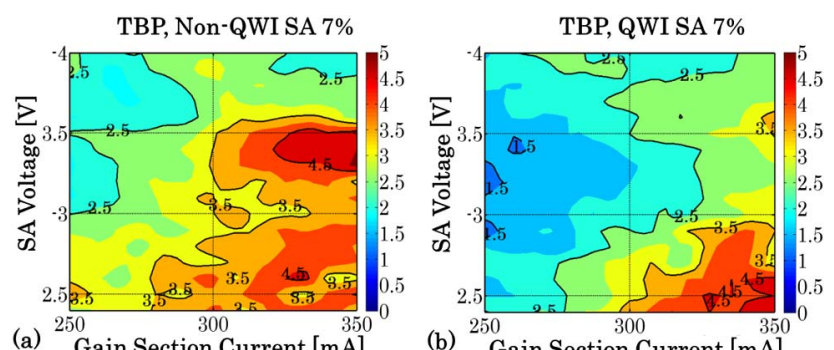

Fig. 6. Color maps show the TBP of the pulses for (a) one SMLL with nonintermixed 7\% SA and (b) a device with a blue-detuned 7\% SA, for currents between 250 and $350 \mathrm{~mA}$.

\section{CONCLUSION}

In summary, we have demonstrated how a bandgap blue detuning applied to the SA of a SMLL can improve the laser performance in terms of pulse width, optical chirp, and peak power. The devices whose absorbers had undergone QWI emit pulses whose FWHM is $30 \%$ lower than that obtained from standard SMLLs fabricated on the same MQW platform. The temporal narrowing exhibited by the pulses also contributed to an improvement in the pulses' peak power, which also experienced an increase between the devices compared. Moreover, the TBP data clearly suggest that a blue-detuned absorber is beneficial in reducing the amount of chirp experienced by the pulses. However, the most interesting feature is the expansion of the region in which stable ML is achieved, with a $\sim 100 \mathrm{~mA}$ current range difference between a detuned absorber and a standard one. The extension of the ML region is in line with the analysis shown in [5], since starting from a shifted SA band edge delays the point where Joule effects become detrimental to the pulse formation mechanism. The narrower pulses and the chirp reduction are thought to be linked to a shorter recovery time exhibited by an intermixed absorber; further experiments will be carried out to confirm this hypothesis.

\section{ACKNOWLEDGMENTS}

The authors acknowledge the support from the technical staff of the James Watt Nanofabrication Centre at Glasgow University and discussions with Salvador Balle. V. Pusino acknowledges partial financial support from the GRPE program and EPSRC under grant agreement EP/P504937/1.

\section{REFERENCES}

1. E. A. Avrutin, J. H. Marsh, and E. L. Portnoi, "Monolithic and multi-gigahertz mode-locked semiconductor lasers: constructions, experiments, models and application," IEE Proc. Optoelectron. 147, 251-278 (2000).

2. D. Burghoff, T. Kao, N. Han, C. I. Chan, X. Cai, Y. Yang, D. J. Hayton, J. Gao, J. L. Reno, and Q. Hu, "Terahertz laser frequency combs," Nat. Photonics 8, 462-467 (2014).

3. Y. L. Kam, C. Harder, J. S. Smith, and A. Yariv, "Passive mode-locking of buried heterostructures lasers with nonuniform current injection," Appl. Phys. Lett. 42, 771-774 (1983).

4. K. Yvind, D. Larsson, L. J. Christiansen, C. Angelo, L. K. Oxenlwe, J. Mrk, D. Birkedal, J. M. Hvam, and J. Hanberg, "Low jitter and high-power 40-GHz all active mode-locked lasers," IEEE Photon. Technol. Lett. 16, 975-977 (2004).

5. J. Javaloyes and S. Balle, "Detuning and thermal effects on the dynamics of passively mode-locked quantum-well lasers," IEEE J. Quantum Electron. 48, 1519-1526 (2012).

6. P. Stolarz, J. Javaloyes, G. Mezósi, L. Hou, C. N. Ironside, M. Sorel, A. C. Bryce, and S. Balle, "Spectral dynamical behavior in passively mode-locked semiconductor lasers," IEEE Photon. J. 3, 1067-1082 (2011).

7. D. Kunimatsu, S. Arahira, Y. Kato, and Y. Ogawa, "Passively mode-locked laser diodes with bandgap-wavelength detuned saturable absorbers," IEEE Photon. Technol. Lett. 11, 13631365 (1999).

8. R. Scollo, H.-J. Lohe, J. F. Holzman, F. Robin, H. Jäckel, D. Erni, W. Vogt, and E. Gini, "Mode-locked laser diode with an ultrafast integrated uni-traveling carrier saturable absorber," Opt. Lett. 30, 2808-2810 (2005).

9. B. S. Ooi, K. Mcllvaney, M. W. Street, A. S. Helmy, S. G. Ayling, A. C. Bryce, J. H. Marsh, and J. S. Roberts, "Selective quantumwell intermixing in GaAs-AlGaAs structures using impurity-free vacancy diffusion," IEEE J. Quantum Electron. 33, 1784-1793 (1997). 
10. L. Hou, M. Haji, J. Akbar, B. Qiu, and A. C. Bryce, "Low divergence angle and low jitter $40 \mathrm{GHz}$ AlGaInAs/InP $1.55 \mu \mathrm{m}$ mode-locked lasers," Opt. Lett. 36, 966-968 (2011).

11. M. J. Strain, P. Stolarz, and M. Sorel, "Passively mode-locked lasers with integrated chirped Bragg grating reflectors," IEEE J. Quantum Electron. 47, 492-499 (2011).
12. J. Javaloyes and S. Balle, "Mode-locking in semiconductor Fabry-Perot lasers," IEEE J. Quantum Electron. 46, 10231030 (2010).

13. C. Huang, H. C. Kapteyn, J. W. McIntosh, and M. M. Murnane, "Generation of transform-limited 32-fs pulses from a selfmode-locked Ti:sapphire laser," Opt. Lett. 17, 139-141 (1992). 